\title{
La riflessione analitica delle funzioni biarmoniche attorno a un cerchio ed alcuni problemi di elasticità piana.
}

\author{
Memoria di L. Sobrero (a Roma).
}

Sunto. - Due funzioni $\mathrm{f}(\mathrm{x}, \mathrm{y})$ e $\varphi(\mathrm{x}, \mathrm{y})$, biamoniche (e cioè soddisfacenti all'equazione $\Delta \Delta=0$ ), rispettivamente definite nei semipiani $\mathrm{x}>0$ ed $\mathrm{x}<0$, le cui derivate seconde si annullano all'infinito, e tali che nei punti dell' asse y risulti $\mathrm{f}=\varphi$ e $\frac{\partial \mathrm{f}}{\partial \mathrm{x}}=\frac{\partial \varphi}{\partial \mathrm{x}}$, si dicono l'una « riflessa》 dell'altra attorno all'asse y. Da ognuna delle due funzioni $\mathrm{f}$ e $\varphi$ l'altra si ottiene con sole operazioni di sostituzione e derivazione (indicando, precisamente, con $\{\mathrm{f}\},\left\{\frac{\partial \mathrm{f}}{\partial \mathrm{x}}\right\}$ e $\{\Delta \mathrm{f}\}$ le funzioni che si ottengono da $\mathrm{f}, \frac{\partial \mathrm{f}}{\partial \mathrm{x}}$ e sf ponendo, in queste, in luogo di $\mathrm{x}$ il suo contrario $-\mathrm{x}$, si ha $\varphi=\{\mathrm{f}\}+2 \mathrm{x}\left\{\frac{\partial \mathrm{f}}{\partial \mathrm{x}}\right\}+\mathrm{x}^{2}\{\Delta \mathrm{f}\}$ e, reci. procamente, $\left.\mathrm{f}=|\varphi|+2 \mathrm{x}\left\{\frac{\partial \varphi}{\partial x}\right\}+\mathrm{x}^{2}|\Delta \varphi|\right)$. In modo analogo si definisce una operazione ditriflessione analitica attorno a un cerchio. La retta potendosi riguardare come cerchio degenere (di raggio infinito) l'operazione di rifiessione analitica attorno alla retta viene ottenuta, nel testo, come caso limite di quella di riflessione attorno al cerchio. $L$ 'operazione di viflessione analitica trova applicazione in alcuni problemi di elasticità piana (perturbazione prodotta da un foro circolare nella sollecitazione di un sistema piano; determinazione degli sforzi in un semipiano elastico sollecitato da una forza applicata in un punto interno).

$\S 1$. Oggetto della ricerca. - Siano $r$ e $\theta$ le coordinate polari nel piano, $f(r, \theta)$ una funzione biarmonica $\left({ }^{1}\right)$ definita nei punti interni al cerchio di raggio unitario $\theta$ centro nell' origine. Se, nell' espressione di $f(r, \theta)$ poniamo, in luogo di $r$, il suo inverso $\frac{1}{r}$, e moltiplichiamo l'espressione così ottenuta per $r^{2}$ otteniamo una nuova funzione $\psi(r, \theta)$, definita nei punti esterni al cerchio unitario, la quale soddisfa ancora, in virtù di un teorema del LeviCrvita, all' equazione $\Delta \Delta=0$. Se conveniamo di indicare con $\{f(r, \theta)\}$ la fun.

(1) e cioè soddisfacente all' equazione $\Delta \Delta f=0$, dove $\Delta$ designa l'operatore di LAPLACE (in coordinate polari: $\Delta=\frac{\partial^{2}}{\partial r^{2}}+\frac{1}{r} \frac{\partial}{\partial r}+\frac{1}{r^{2}} \frac{\partial^{2}}{\partial \theta^{2}}$ ). 
zione che si ottiene da $f(r, \theta)$ ponendo in luogo di $r$ il suo inverso $\frac{1}{r}$, avremo (in simboli)

$$
\psi(r, \theta)=r^{2}\{f(r, \theta)\}\left({ }^{1}\right)
$$

Si constata che nei punti del cerchio unitario le due funzioni $f(r, \theta) \theta$ $\psi(r, \theta)$ assumono gli stessi valori, mentre assumono valori generalmente differenti le loro derivate normali $\left({ }^{2}\right)$.

Un problema di elastieità del quale in seguito faremo cenno ci ha condotti a ricercare l'operazione (di tipo analogo a quella che fa passare dalla funzione $f$ alla funzione $\psi$ ) la quale muta un'assegnata funzione biarmonica $f(r, \theta)$ (definita nei punti interni al cerchio unitario) in una nuova funzione biarmonica $\varphi(r, \theta)$, definita nei punti esterni al cerchio unitario, di cui si aunullano all'infinito le derivate seconde rispetto ad $r$, e tale (infine) che essa e la sua derivata normale rispettivamente eguaglino, nei punti del cerchio unitario, la funzione $f$ e la sua derivata normale. Proveremo nel prossimo paragrafo che ogni funzione $\varphi(r, \theta)$ soddisfacente a queste condizioni è fornita dalla formula:

$$
\begin{gathered}
\varphi=\{f\}+\left(r-\frac{1}{r}\right)\left\{\frac{\partial f}{\partial r}\right\}+\frac{1}{4}\left(r-\frac{1}{r}\right)^{2}\{\Delta f\}+ \\
+\left[\frac{1}{4}(\Delta f)_{r=0}\left(1+2 \log r-r^{2}\right)+(a \cos \theta+b \sin \theta)\left(\frac{1}{r}+2 r \log r-r\right)\right],
\end{gathered}
$$

(1) Si veda in proposito: T. Levi-Crvita, Sopra una trasformazione in sè stessa dell'equazione $\underset{2 \nexists z}{\Delta}=0$, "Atti del $R$. Istituto Veneto di scienze, lettere ed arti", To. IX, Ser. VII, 1897-8, pp. 1399-1410. La proprietá dimostrata dal LEvi-Crvira immediatamente si estende alle funzioni m-armoniche di ordine qualunque: se $\mathrm{f}(\mathrm{r}, \theta)$ è una funzione $\mathrm{m}$-ar* monica (e cioè soddisfacente all' equazione $\Delta \mathrm{mf}=0$, dove $\Delta \mathrm{m}$ designa l'operatore $\Delta$ ripetuto $\mathrm{m}$ volte) anche la funzione $\psi=\mathrm{r}^{2}(\mathrm{~m}-1)$ f $\}$ risulta $\mathrm{m}$-armonica. Ciò si vede, nel miglior modo, ricordando (con E. Almaxs, Sull integrazione dell'equazione $\Delta 2 \mathrm{n}=0$, "Annali di Matematica , Ser. III, To. II, 1899, pp. 1-51) che ogni funzione m-nrmonica $f(r, 0)$ definita nei punti di un' area monoconnessa può sempre scomporsi in una somma del tipo

$$
f_{0}+r^{2} f_{1}+\ldots+r^{2(m-1)} f_{m-1} \text {, }
$$

dove $f_{0}, f_{1}, \ldots, f_{m-1}$ sono funzioni armoniche. Segue immediatamente:

$$
\psi=r^{2(m-1)}\left|f_{0}\right|+v^{2(m-2)}\left|f_{1}\right|+\ldots+\left|f_{n i z-1}\right| \text {. }
$$

Poichè $\left.\left\{f_{0}\right\},\left|f_{1}\right|, \ldots, \mid f_{m-1}\right\}$ sono ancora notoriamente funzioni armoniche il secondo membro della precedente relazione è una somma del tipo (*), e quindi funzione m-armonica.

(2) Per derivate normali intenderemo quelle eseguite in direzione normale al cerchio unitario, e cioè le derivate rispetto ad $r$. 
dove: ... $\{f\},\left\{\frac{\partial f}{\partial r}\right\}$ e $\{\Delta f\}$ sono le funzioni che rispettivamente si ottengono da $f, \frac{\partial f}{\partial r}$ e $\Delta f$ ponendo, in tali espressioni, in luogo di $r$ il suo inverso $\frac{1}{r}$;

$\ldots(\Delta f)_{r=0}$ è il valore di $\Delta f$ calcolato nell' origine del riferimento;

$\ldots a$ e $b$ sono coefficenti arbitrari. Tutte le funzioni (1), qualunque sia il valore dei coefficenti $a$ e $b$, soddisfano al problema proposto. L'operazione che muta l'assegnata funzione biarmonica $f(r, \theta)$ nella funzione $\varphi(r, \theta)$ si dirà di «riflessione analitica» attorno al cerchio unitario; la funzione $\varphi(r, \theta)$ prenderà il nome di « funzione riflessa» $\left.{ }^{1}\right)$.

§ 2. Esame della funzione riflessa. - Proviamo, anzitutto, che il secondo membro della (1) è funzione biarmonica. Poichè son (notoriamente) biarmonici i termini contenuti entro parentesi quadre, basterà dimostrare che è funzione biarmonica la somma

$$
\{f\}+\left(r-\frac{1}{r}\right)\left\{\frac{\partial f}{\partial r}\right\}+\frac{1}{4}\left(r-\frac{1}{r}\right)^{2}\{\Delta f\} .
$$

Il modo più comodo per convincersene consiste nello scomporre la funzione $f$ in una somma del tipo

$$
f=f_{0}+r^{2} f_{1}\left({ }^{2}\right)
$$

$f_{0}$ ed $f_{1}$ essendo funzioni armoniche. Si vede allora con breve calcolo che l'espressione (2) assume l'aspetto:

$$
\left\{f_{0}-r \frac{\partial f_{0}}{\partial r}-r \frac{\partial f_{1}}{\partial r}\right\}+r^{2}\left\{f_{1}+r \frac{\partial f_{0}}{\partial r}+r \frac{\partial f_{1}}{\partial r}\right\} .
$$

Poichè $f_{n}, f_{1}, r \frac{\partial f_{0}}{\partial r}, r \frac{\partial f_{1}}{\partial r}$ sono funzioni armoniche, tali risultano anche le espressioni $\left\{f_{0} \quad r \frac{\partial f_{0}}{\partial r}-r \frac{\partial f_{1}}{\partial r}\right\}$ ed $\left\{f_{1}+r \frac{\partial f_{0}}{\partial r}+r \frac{\partial f_{1}}{\partial r}\right\}$. La somma (4) è dunque del tipo (3), e quindi funzione biarmonica.

Nessuna difficolta s'incontrerebbe a provare che il secondo membro della (1) verifica le condizioni al contorno richieste per la funzione $\varphi$; e che tutte le funzioni $\varphi$ soddisfacenti al problema si ottengono colla formula (1).

(1) Per una analoga formula di transizione da "problemi interni " a «problemi esterni » (nel caso di funzioni armoniche) si veda: C. A. BJerknes, Hydrodynamische Fernkräfte, "Ostwald's Klassiker der exakten Wissenschaften" (N. 195), p. 101.

(2) Cfr. la forma di scrittura $\left(^{*}\right)$ nella nota $\left({ }^{2}\right)$ della pagina precedente. 
Aggiungeremo che alla formula (1) siam pervenuti cereando, per la funzione $\varphi(r, \theta)$, uno sviluppo in serie. I relativi calcoli, banali e laboriosi, non offrono alcun interesse. Il fatto che le serie ottenute col procedimento in. dicato si possano sommare cosi da ottenere la formula finita (1), lascia tuttavia sospettare che si debba poter giungere a questa formula con altro più adeguato procedimento.

\$ 3. Il problema della piastra forata. - Della formula (1) faremo uso nella soluzione del seguente problema: «Un sistema elastico piano indefinito $\grave{e}$ soggetto a un insieme di forze esterne in equilibrio agenti nel suo medesimo piano. In una regione nella quale non cadono punti d'applicazione di forze esterne si pratica un foro circolare. Con ciò viene evidentemente ad alterarsi lo stato di sollecitazione elastica del sistema. Qual'è l'entità di questa perturbazione? $\gg(')$.

Immediata è la traduzione analitica del problema. Prima che si pratichi il foro lo stato di sollecitazione elastica o caratterizzato da una certa funzione di ArRy $f(r, \theta)\left({ }^{2}\right)$. Praticato nella piastra nn foro circolare (che potremo supporre di centro nell' origine e raggio unitario) verrà ad alterarsi lo stato di sollecitazione elastica del sistema. Il nuovo stato di equilibrio sarà carat. terizzato da una nuova funzione di AIRY $F(r, \theta)$ (definita nei punti esterni al cerchio unitario) la quale potrà riguardarsi come somma dell'antica funzione $f(r, \theta)$ e di una funzione, che potremmo dire aggiuntiva, $\varphi(r, \theta)$. Si vede facilmente che tale funzione aggiuntiva $\varphi(r, \theta)$, la quale sta a rappresentare la perturbazione elastica prodotta dalla presenza del foro, risulta soggetta alle seguenti condizioni:

(1) Il problema non è nuovo. Posto e risolto (empiricamonte) dal KIRsch in un caso particolare (Die Theorie der Elastizität und die Bediurfnisse der Festigkeitslehre, "Zeit. des Vereins Deut. Ing. ", Band 42, 1892, n. 29, p. 797) fu, in seguito, razionalmente ripreso dal WoLf (Beiträge zur ebenen Elastizitätstheorie, «Zeit, für techn. Physik », 1921, Heft. 8, p. 29; 1922, Heft. 5, p. 160), dal Pöschr e da altri. Il metodo di soluzione che noi qui proponiamo si differenzia sia per la semplicità del mezzo analitico eni fa ricorso, sia perohè porge una formula risolutiva unica, indipendentemente dalla natura dello sforzo che sollecita la piastra in assenza di foro.

$\left({ }^{2}\right)$ E ben noto che le derivate seconde (pure e mista) della funzione $f$, secondo due direzioni ortogonali $x$ ed $y$, rappresentano (a meno di un segno) le componenti della sollecitazione elastica secondo quelle direzioni. Si ha precisamente, indicando con $\sigma_{x}, \sigma_{y}$ e $\tau$ le componenti della sollecitazione elastica,:

$$
\sigma_{x}=\frac{\partial^{2} f}{\partial y^{2}}, \quad c_{y}=\frac{\partial^{2} f}{\partial x^{2}}, \quad \tau=-\frac{\tau^{2} f}{\partial x \partial y} .
$$

Queste formule possono, reciprocamente, servire come definizione della funzione $f$. 
1) Essa verifica, in tutti i punti dove è definita (e ciò̀ nei punti esterni al cerchio unitario) l'equazione $\Delta \Delta \varphi=0$.

2) Dovendosi ritenere che la sollecitazione «aggiuntiva» dovuta alla presenza del foro si avverta solo nella immediata prossimità di quest'ultimo (e cioè abbia carattere nettamente locale), la derivata $\frac{\partial^{2} \varphi}{\partial r^{2}}$ (che appunto rap* presenta una delle componenti della sollecitazione elastica aggiuntiva) dovrà tendere a zero al crescere di $r$ ad infinito.

3) Si deduce infine dalla teoria della funzione di AIRx ehe la funzione $F(r, \theta)$ e la sua derivata prima rispetto ad $r$ devono annullarsi lungo il bordo del foro (1). E poichè $F=f+\varphi$ si trae che dovrà essere (nei punti del cerchio unitario) $\varphi=-f$ e $\frac{\partial \varphi}{\partial r}=-\frac{\partial f}{\partial r}$.

(1) Che sia così appare quasi immediato se si ricorda il significato meccanico della funzione di AIRY (cfr. L. Sorrero, Del significato meccanico della funzinne di Airy, "Ren. diconti della R. Ace. dei Lincei », 1935, fascicolo 4; e « Ricerche d'Ingegneria », 1935). Sia un sistema elastico piano, eventualmente pluriconnesso (v. figura 1), privo di torze superficiali e sollecitato da forze esterne soltanto nei punti del suo contorno esterno $E$. Nel piano del sistema siano: $O$ un punto fisso (p. es. l'origine del riferimento), $P$ un punto generico di coordinate $x$ ed $y, C$ una curva con l'origine in $O$ e l'estremo in $P$. Supporremo che tale curva (e con essa gli estremi $O$ e $P$ ) sia traeciata completamente all'interno del contorno $E$ del sistema piano; ma ammetteremo ehe alcuni tratti della curva (ed anche $i$ suoi punti terminali) possano eventual. mente cadere nell'interno delle cavità del sistema. ì facile constatare che il momento $M$, preso rispetto al punto $P$,

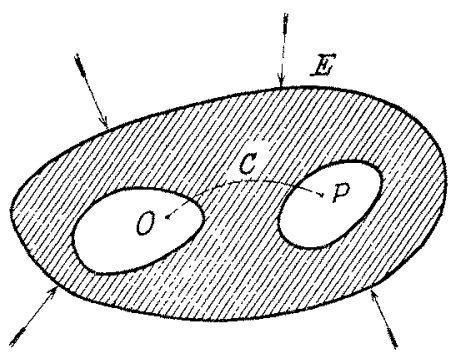

Fig. 1 degli fforai che la parte di sistema situata alla destra della curva $C$ esercita (attraverso tale curva) sulla parte di sistema situata alla sinistra, non dipende dall' andamento della enrva $C$, ma solo dall' estremo (variabile) $P$ di tale curva. Quando, inoltre, il punto $P$ cade nell'interno del sistema elastico, il momento $\mathrm{M}$ coincide col valore, nel punto $\mathrm{P}$, della funzione di Airy (della quale, pertanto, fornisce l' interpretazione meccanica). Si noti che la funzione $M(x, y)$ conserva significato, contrariæmente alla funzione di ArRY, anche nei punti interni alle cavità del sistema.

Se il punto $P(x, y)$ si muove entro una di queste cavità, la funzione $M(x, y)$ varia con legge lineare (in $x$ ed $y$ ); se il punto $P$, uscendo da una cavità, entra nel sistema piano, le derivate seconde, terze,... della funzione $M(x, y)$ subiscono una brusca variazione, ma la fnnzione stessa e le sue derivate prime variano con legge continua.

Nel sistema piano cui si riferisce il testo (sistema indefinito con foro circolare unico) si prenda come punto fisso $O$ il centro del foro. E allora manifesto che la funzione $M$ e le sue derivate prime si mantengono nulle in tutti $i$ punti interni al foro, Dalla proprieta di continuità di $M$ e delle sue derivate prime si trae inoltre che, se il punto $P$ è situato sul sistema piano e si sposta verso un punto qualunque del bordo del foro, la funzione $M$ (e cioè la funzione di ArRY) e le sue derivate prime tendono a zero. Di qui la condizione al contorno espressa nel testo. 
Ma una funzione $\varphi(r, \theta)$ soddisfacente alle condizioni ora dette differisce solo per il segno da quella ottenibile da $f(r, \theta)$ con l'operazione di riflessione analitica attorno al cerchio unitario. Sicchè risulterà, per quanto si è visto nel precedente $\$ 1$ :

$$
\begin{gathered}
\varphi(r, \theta)=-\{f\}-\left(r-\frac{1}{r}\right)\left\{\frac{\partial f}{\partial r}\right\}-\frac{1}{4}\left(r-\frac{1}{r}\right)^{2}\{\Delta f\}- \\
-\left[\frac{1}{4}(\Delta f)_{r=0}\left(1+2 \log r-r^{2}\right)+(a \cos \theta+b \sin \theta)\left(\frac{1}{r}+2 r \log r-r\right)\right] .
\end{gathered}
$$

I coefficienti $a$ e $b$ co iparenti in questa formula non risultano determinabili con le condizioni fi ora imposte alla funzione $\varphi(r, \theta)$. Ma è noto, d'altra parte, che negli sviluppi in serie della funzione di ArRx devono mancare $\mathbf{i}$ termini in $\cos \theta \cdot r \log r \in \mathcal{L}$ in $\sin \theta \cdot r \log r$ se si vuole conservare la monodromia degli spostamenti $\left.{ }^{(}\right)$. Dovrà dunque risultare, nello sviluppo soprascritto, $a=b=0$, e la funzione $F(r, \theta)$ arrà l'espressione che segue:

$$
\begin{gathered}
F(r, \theta)=f(r, \theta)+\varphi(r, \theta)= \\
=f-\{f\} \quad\left(r-\frac{1}{r}\right)\left\{\frac{\partial f}{\partial r}\right\}-\frac{1}{4}\left(r-\frac{1}{r}\right)^{2}\{\Delta f\}-\frac{1}{4}(\Delta f)_{r=0}\left(1+2 \log r-r^{2}\right) .
\end{gathered}
$$

La formula (5) risolve compiutamente il problema proposto.

\& 4. Piastra forata: casi particolari. - Supponiamo, in primo luogo, che la piastra risulti sollecitata a trazione pura (cosicchè, scegliendo come asse delle $x$ la direzione di trazione, risulti, in assenza di foro: $\sigma_{x}=p$, $\sigma_{y}=\tau=0, p$ essendo una costante). Avremo allora (assunto un riferimento polare in eui l'asse coincida coll' asse $x$ ):

$$
f=\frac{1}{2} y^{2} p=\frac{1}{2} r^{2} \sin ^{2} \theta \cdot p
$$

e quindi, per la (5),

$$
F^{\prime}=\left[\left(\frac{r^{2}}{4}-\frac{1}{2} \log r-\frac{1}{4}\right)-\frac{1}{2}\left(r-\frac{1}{r}\right)^{2} \cos 2 \theta\right] p .
$$

Indicando pertanto con $\sigma_{v}, \sigma_{t}, \tau, \cdot$, le componenti della sollecitazione elastica della piastra forata nella direzione del raggio vettore e della sua

(1) Si confronti: L. Sobreno, Contributo alla teoria della funzione di Airy, « Memorie della R. Acc. d'Italia ", Roma, 1935. 
normale (orientata nel senso delle $\theta$ crescenti) si avrà:

$$
\left\{\begin{aligned}
\sigma_{r}=\frac{1}{r} \frac{\partial F}{\partial r}+\frac{1}{r^{2}} \partial \partial^{2} F & =\left[\frac{1}{2}\left(1-\frac{1}{r^{2}}\right)\left(1+\left[1-\frac{3}{r^{2}}\right] \cos 2 \theta\right)\right] p, \\
\sigma_{t}=\frac{\partial^{2} F}{\partial r^{2}} & =\left[\frac{1}{2}\left(1+\frac{1}{r^{2}}\right)-\frac{1}{2}\left(1+\frac{3}{r^{4}}\right) \cos 2 \theta\right] p, \\
\tau_{r, t}=-\frac{1}{r} \frac{\partial^{2} F}{\partial r \partial \theta}+\frac{1}{r^{2}} \frac{\partial F}{\partial \theta} & =\left[-\frac{1}{2}\left(1-\frac{1}{r^{2}}\right)\left(1+\frac{3}{r^{2}}\right) \sin 2 \theta\right] p,
\end{aligned}\right.
$$

(le quali naturalmente coincidono con quelle indicate dal KInsch).

Consideriamo, in secondo luogo, il caso di un sistema piano indefinito sollecitato da uno sforzo tagliante di entità $t$ (cos̀̀ che risulti, in assenza di foro, $\sigma_{x}=\sigma_{y}=0, \tau=t$, dove $t$ è una costante). Avremo

$$
f=-x y t=-\frac{1}{2} r^{2} \sin 2 \theta \cdot t,
$$

e quindi, per la formula (5),

da cui :

$$
F=-\frac{1}{2}\left(r-\frac{1}{r}\right)^{2} \sin 2 \theta \cdot t
$$

$$
\left\{\begin{aligned}
\sigma_{r} & =\left(1-\frac{1}{r^{2}}\right)\left(1-\frac{3}{r^{2}}\right) \sin 2 \theta \cdot t, \\
\sigma_{t} & =-\left(1+\frac{3}{r^{4}}\right) \sin 2 \theta \cdot t, \\
\tau_{r, s} & =\left(1-\frac{1}{r^{2}}\right)\left(1+\frac{3}{r^{2}}\right) \cos 2 \theta \cdot t\left({ }^{1}\right) .
\end{aligned}\right.
$$

\$. 5. La riflessione analitica attorno a una retta. - Sia $f(x, y)$ una funzione biarmonica delle variabili $x$ ed $y$, definita nei punti del semipiano $x>0$. Se, nell' espressione di $f(x, y)$, poniamo in luogo di $x$ il suo contrario - $x$, otteniamo una nuova funzione, anch'essa biarmonica, definita nei punti del semipiano $x<0$. Designamo questa nuova funzione col simbolo $\{f\}$. E facile constatare che, nei punti dell'asse $y$, le due funzioni $f$ ed $\{f\}$ assumono i medesimi valori; mentre assumono valori generalmente differenti le loro derivate rispetto ad $x$.

(1) Si confronti: L. SoBRero, Algebra delle funzioni ipercomplesse e sue applicazioni alla teoria matematica dell' elasticità, "Memorie della R. Ace. d'Italia", Roma, 1984, p. 44; idem, Theorie der ebenen Elastizität unter Benutzung eines Systens hyperhomplexer Zahlen, "Hamburger mathematisehe Einzelschriften ", Teubner, Leipzig, 1934. 
Seguendo l'indirizzo già indicato nei precedenti paragrafi abbiam cercata un'operazione (di tipo analogo a quella che muta la funzione $f$ nella funzione $\{f\}$ ), la quale faccia passare da una (data) funzione biarmonica $f(x, y)$, definita nel semipiano $x>0$, e tale che le sue derivate seconde si annullino all' infinito, ad una nuova funzione biarmonica $\varphi(x, y)$, definita nei punti del semipiano $x<0$, ed ivi soddisfacente alle seguenti condizioni al contorno: che le sue derivate seconde si annullino all'infinito, e che essa e le sue derivate parziali prime assumano, nei punti dell'asse $y$, rispettivamente gli stessi valori della funzione $f$ e delle sue derivate parziali prime.

Ad una siffatta operazione daremo il nome di «riflessione analitica » attorno ad una retta. Potendosi, la retta, riguardare come un cerchio degenere (di raggio infinito) l'operazione di riflessione attorno alla retta risulta come caso limite di quella di riflessione attorno a un cerchio. Eseguendo l'indicato passaggio al limite sulla formula (1) si vede che la richiesta funzione $\varphi$ risulta espressa dalla relazione

$$
\varphi=\{f\}+2 x\left\{\frac{\partial f}{\partial x}\right\}+x^{2}\{\Delta f\}
$$

dove con le notazioni $\{f\},\left\{\frac{\partial f}{\partial x}\right\}$ e $\{\Delta f\}$ abbiamo indicate le funzioni che da $f, \frac{\partial f}{\partial x}$ e $\Delta f$ rispettivamente si ottengono, ponendo in tali espressioni in luogo di $x$ il suo contrario - $x$.

$\grave{\mathrm{E}}$ facile constatare che $i l$ quadrato dell'operazione di riflessione analitica attorno alla retta è l'identità. Con ciò intendendo affermare che se si applica alla funzione riflessa $\varphi(x, y)$ l'operazione di riflessione analitica, si ritorna alla funzione primitiva $f(x, y)$. In simboli:

$$
f=\{\varphi\}+2 x\left\{\frac{\partial \varphi}{\partial x}\right\}+x^{2}\{\Delta \varphi\}\left({ }^{1}\right) \text {. }
$$

\$6. Il problema del semipiano elastico. - Faremo applicazione della formula (6) al seguente problema di elasticità piana: «Un sistema piano indefinito è soggetto a un insieme di forze esterne agenti nel suo medesimo piano, ed applicate in punti del semipiano (che diremo positivo) $x>0$. Se si

(1) Riteniamo che operazioni di "riflessione analitica" analoghe a quelle indicate nel testo sussistano per altri tipi di funzioni (diverse dalle biamoniche) e per altri tipi di curve (diverse dal cerchio e dalla retta). 
taglia il piano elastico Iungo l'asse delle $x$ viene manifestamente ad alterarsi lo stato di sollecitazione del sistema. Qual'è l'entità di questa perturbazione?" ».

Indichiamo con $f(x, y)$ la funzione di AIRY che definisce lo stato di sollecitazione elastica del sistema piano prima del taglio; con $F(x, y)$ la funzione che individua la sollecitazione elastica del piano tagliato.

A taglio avvenuto il semipiano negativo $(x<0)$ viene a formare un sistema a sè nel quale mancano forze esterne direttamente applicate; talchè risulterà (in tutti $\mathrm{i}$ punti di questo semipiano negativo) $\sigma_{x}=\sigma_{y}=\tau=0$, e quindi $F(x, y) \equiv 0$.

Per quanto, invece, riguarda il semipiano positivo, si può dimostrare (con ragionamento analogo a quello svolto nel $\$ 3$ ) che la funzione $F(x, y)$ ivi risulta come differenza tra la funzione $f(x, y)$ e la funzione $\varphi(x, y)$ ottenuta per riflessione di $f(x, y)$ attorno all'asse delle $y$. Si ha cioè:

$$
F(x, y)=f-\{f\}-2 x\left\{\frac{\partial f}{\partial x}\right\}-x^{2}\{\Delta f\},
$$

la quale risolve compintamente il problema.

\$ 7. Semipiano clastico: caso particolare. - In applicazione alla formula (7) proponiamoci, a titolo di esempio, di determinare la sollecitazione prodotta nel semipiano elastico $x>0$ (con il bordo $x=0$ libero da forze esterne) da una forza di entità $P$, diretta secondo l'asse $x$, ed applicata nel punto di coordinate $(1,0)$ (v. figura 2) (1).

Nel piano indefinito lo stato di sollecitazione elastica prodotto dalla forza $P$ risulta espresso dalla funzione:

$$
f(x, y)=\frac{P}{\pi}\left(\frac{1}{2} y^{\theta}-\frac{m-1}{4 m}[x-1] \log r\right)
$$

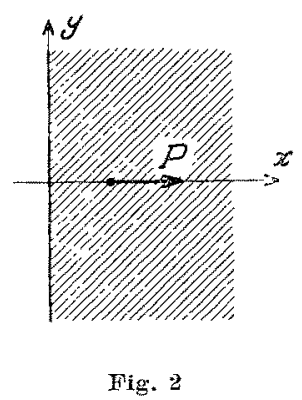

dove si ̀̀ indicato con $m$ il rapporto degli allungamenti alle contrazioni laterali, e si ̀̀ posto $\theta=\operatorname{arctang} \frac{y}{x-1}, r=\sqrt{y^{2}+(x-1)^{z}}$.

Nel semipiano elastico la funzione $F(r, \theta)$ che definisce lo stato di sollecitazione provocato dalla forza $P$, si otterrà dalla formula (7) tenendo conto

(1) Il problema è stato posto ed esaminato da E. MeLAx, Der Spannungszustand der durch eine Einzelkraft im Innern beanspruchten Halbscheibe, "Zeit. fur angew. Math. und Mech. ", Band 12, Heft. 6. Dic. 1932, pp. 343-346. 
dell' espressione (8) di $f(x, y)$. Con breve calcolo si ha:

$$
F=\frac{P}{\pi}\left(\frac{1}{2} y\left[\theta+\theta^{\prime}\right]-\frac{m-1}{4 m}[x-1] \log \frac{r}{r^{\prime}}-\frac{m+1}{2 m} \frac{x(x+1)}{r^{\prime 2}}+x\right),
$$

dove si è posto $\theta^{\prime}=\operatorname{arctang} \frac{y}{x+1}, r^{\prime}=\sqrt{y^{2}+(x+1)^{2}}$. La funzione $F$ ora determinata coincide, sostanzialmente, con quella indicata dal MELAN (1).

(1) Nella formula del MéLAN non figura l'ultimo termine, $\frac{P}{\pi} x$, della nostra formula. Ma tale differenza non porta evidentemente ad alcun divario nell'espressione degli sforzi (che si ottengono come derivate seconde della $F$ ). 